\title{
A Comparison of Joint ICA and Parallel ICA Multimodal Fusion Methods in Schizophrenia
}

\author{
Abdullah N. Mohammed \\ Faculty of Electronic \\ Engineering \\ Menofia University \\ Menouf, Egypt
}

\author{
Taha E. Taha \\ Faculty of Electronic \\ Engineering \\ Menofia University \\ Menouf, Egypt
}

\author{
Osama S. Faragallah \\ Faculty of Electronic \\ Engineering \\ Menofia University \\ Menouf, Egypt
}

\begin{abstract}
Schizophrenia is still considered unknown disease that needs more study and analysis. In this case Multimodal fusion is a good way to make analysis on the joint information found on the different imaging modalities related to this disease. This paper discusses Schizophrenia analysis using two approaches of the common Brian Imaging multimodal fusion approaches (Joint ICA and Parallel ICA). The aim of this study is to investigate these two approaches for more understanding showing their strengths, limitations, and analysis strategies.
\end{abstract}

\section{General Terms}

Medical image fusion

\section{Keywords}

Image fusion, Joint ICA, Parallel ICA, Schizophrenia, fMRI image

\section{INTRODUCTION}

Schizophrenia is a chronic disease that impairs a lot of cognitive domains such as memory, attention, and executive functions [1]. A hypothesis stating that, a misconnection of neural circuitry in some networks of the brain regions (or dysfunctional integration between them) would be one of the causes of the schizophrenia [1]. Due to brain data can be collected from a various imaging techniques, which are varying from each other. For example; Functional Magnetic Resonance Imaging (fMRI) shows the hemodynamic response related to the neural activity in the brain. Structural Magnetic Resonance Imaging (sMRI) gives information about the brain tissue type in a definite position [2]. Diffusion tensor imaging (DTI) provides information about the brain networks connections [3]. Electroencephalography (EEG) measures the brain electrical activity with a high temporal resolution (higher than fMRI) and low spatial resolution [4]. These imaging techniques provide a unique and very important characteristic of the brain, and all of them together can provide a good amount of information about the brain. So a lot of studies had been published trying to reveal the neural mechanisms of schizophrenia using these different brain imaging modalities (fMRI [5, 6], sMRI [7, 8], DTI [9, 10], and genetics [11, 12]).

Unfortunately, Schizophrenia is one of the most mental disorders that cause human suffering. In Schizophrenic it was noticed that hallucinations in the auditory and visual modalities are the most frequent experiences [13, 14]. With imaging the brain activity of those patients it was noticed that the brain activity in speech production is increased [15]. In spite of knowing that there is a relation between (functional, structural, and genetic abnormalities in the brain) and this disease, but till now there is not enough information that can help in making a clinical decision. All the diagnosis applied on the brain in this case is just done for symptom assessment [1]. One of the solutions to overcome this problem and having more information about the disease is to analyze the different imaging modalities together, by combining them using the multimodal fusion techniques.

The following sections of the paper show two methods of the multimodal fusion methods (Joint Independent Component Analysis, and Parallel Independent Component Analysis). The two methods enable us to analyze the schizophrenia (brain functional, structural, genetic aspects) for more understanding about it. The paper also will discuss their strengths, limitations and their analysis strategies.

\section{JOINT INDEPENDENT COMPONENT ANALYSIS (Joint ICA)}

The need of the joint analysis methods became important to enable examining and analyzing the shared information between the features found in the different imaging modalities to be fused. One of these methods is the Joint ICA.

Joint ICA is applied on two different imaging modalities and extracts the spatially independent maps for each modality of them. Usually these maps are coupled together by a shared loading parameter. Joint ICA have been used in fusing couples of the modalities (fMRI-sMRI (GM) [16-18], fMRIEEG [19-22], fMRI-DTI (FA) [23, 24], GM-WM [25]). In this paper Joint ICA was applied on two modalities fMRI and EEG of both healthy and Schizophrenia patients to jointly compare the features of each of them together to find some features that can describe the nature of the Schizophrenia.

Joint ICA assumes that the two or more modalities have the same mixing matrix [26].

The basic steps of the Joint ICA (as it was implemented in the Fusion ICA Toolbox (FIT)) can be showed in Fig.1, and summarized as following [27]:

The features of each imaging modality are computed and collected.

For each task a normalization process is done on the collected features.

Principal Component Analysis (PCA) is used in reducing the dimensions of the normalized features.

The spatially independent Components are extracted from the reduced data obtained by PCA, and as noted each component will share a common loading or mixing parameter between the tasks. 


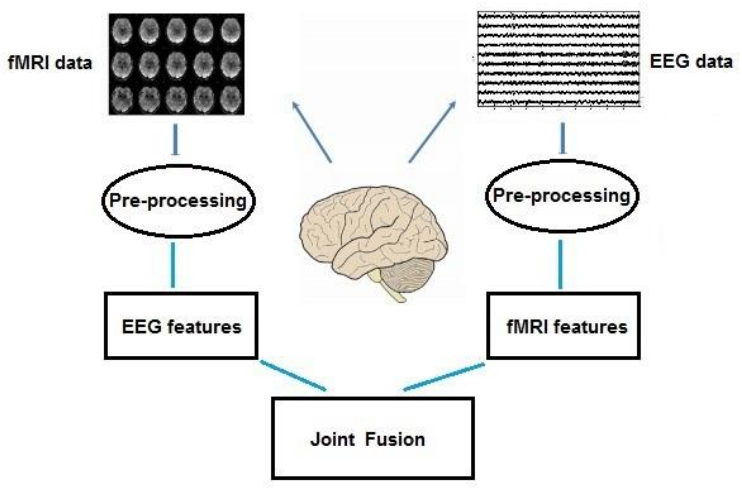

Fig 1: fMRI-EEG fusion steps using Joint ICA.

Joint ICA fusion method has the following advantages; first, it can be used in identifying some diseases such as Schizophrenia (SZ), and that is due to the activation patterns that can be obtained from different brains images that are SZ patients will be similar in multiple tasks. Second, Joint ICA shows a good spatial and temporal resolution in case of fusing fMRI, and EEG modalities together, and that is because fMRI has a good spatial resolution and EEG has a good temporal resolution.

As shown in Fig.2. The figure is divided to three parts. Part A shows the loading parameters of each control, patient and the component. Part B shows fMRI image for the temporal and frontal lobe regions of the brain. Part $\mathrm{C}$ shows another example of applying the Joint ICA on GM/WM to have more joint features about the brain [28].

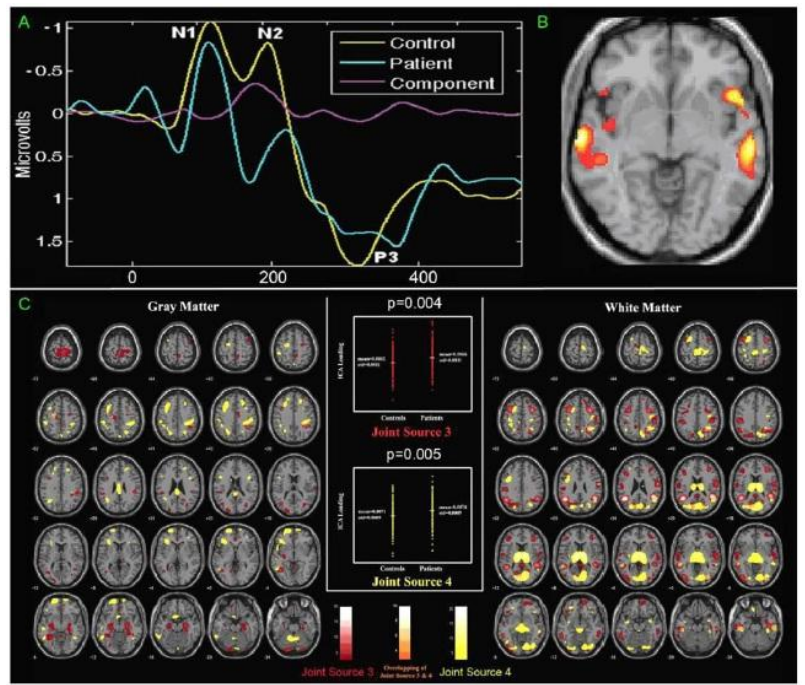

Fig 2: Fusion of the fMRI and EEG modalities using Joint ICA.

\section{PARALLEL INDEPENDENT COMPONENT ANALYSIS (Parallel ICA)}

Parallel ICA is an extension of the ICA that is used in analyzing multiple modalities. As it was mentioned previously Joint ICA takes into consideration the whole inter effects between the two modalities to be fused. Parallel ICA finds the hidden factors from both modalities and the connections between them by finding the independent components of both the modalities and their connections [29]. Parallel ICA had been used in fusing multiple modalities such as fMRI-EEG $[30,31]$, fMRI-Gene (SNP) [32, 33], GM-Gene (SNP) [34, $35]$.
Both Joint ICA and Parallel ICA are depending on the shared mixing matrix, but Parallel ICA assumes that the two data sets are mixed in a similar pattern but not identical one such as Joint ICA. Parallel ICA is more interested and focusing on the individual linked components and their connections [36].

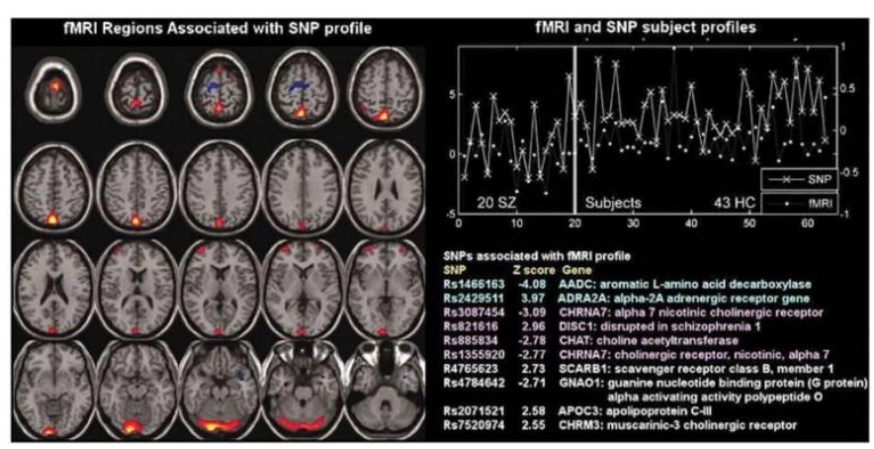

Fig 3: Fusion of the fMRI and SNP modalities using Parallel ICA.

Fig.3 shows using the parallel ICA fusion method in the analysis of fMRI data and 367 SNPs from 20 SZ patients and 43 healthy controls [29]. As shown Parallel ICA divides the output to three regions, the left one represent the fMRI part, the lower right part represent the SNP detailed part, and the upper right part which represent subject profiles obtained from fusing fMRI and SNP parts together.

\section{SUMMARY}

Finally it can be concluded that, Joint analysis is very important issue in case of the need of analyzing the shared information between the features found in different modalities.

Joint ICA and Parallel ICA are two of the most common Joint analysis techniques, which can be used in fusing two imaging modalities to analyze the shared information and features between them. According to previous studies Joint ICA is very efficient in case of analyzing fMRI and EEG modalities together; in this case it assumes sharing the same mixing matrix between the features of the two modalities.

Parallel ICA also can be used between any two modalities, but it was widely used in analyzing fMRI and Genetic data by fusing them together.

Analyzing of medical images using one of the last methods can help in understanding and knowing more about Schizophrenia features, which may help in early prediction of it by comparing any new features of any suspected case with the previous known features of Schizophrenia which can help clinical decision makers in taking the appropriate action towards this disease.

\section{ACKNOWLEDGMENTS}

Thanks to our supervisors and many persons who have contributed in the advance of this work.

\section{REFERENCES}

[1] Jing Sui, Qingbao Yu, Hao He, Godfrey D. Pearlson, and Vince D. Calhoun.2012. A Selective review of multimodal fusion methods in schizophrenia. Human Neuroscience.

[2] Robert W. McCarley, Cynthia G. Wible, Melissa Frumin, Yoshio Hirayasu, James J. Levitt, Iris A. Fischer, and 
Martha E. Shenton. 1999. MRI Anatomy of Schizophrenia. Biol Psychiatry.

[3] Sui J, Pearlson GD, Caprihan A, Adali T, Kiehl KA, et al. 2011. Discriminating Schizophrenia and Bipolar Disorder by Fusing FMRI and DTI in A Multimodal CCA+ Joint ICA Model. Neuroimage.

[4] B. He, and Z. Liu. 2008. Multimodal Functional Neuroimaging: Integrating Functional MRI and EEG/MEG. In Proceedings of IEEE Rev Biomed Eng.

[5] Pearlson, G. D. 1997. Superior temporal gyrus and planum temporale in schizophrenia: a selective review. Biol. Psychiatry.

[6] Loeber, R. T., Sherwood, A. R., Renshaw, P. F., Cohen, B. M., and YurgelunTodd, D. A. 1999. Differences in cerebellar blood volume in schizophrenia and bipolar disorder. Schizophr.

[7] Giuliani, N. R., Calhoun,V. D., Pearlson, G. D., Francis, A., and Buchanan, R. W. 2005. Voxel-based morphometry versus region of interest: a comparison of two methods for analyzing gray matter differences in schizophrenia. Schizophr.

[8] Douaud, G. Smith, S. Jenkinson, M. Behrens, T. Johansen-Berg, H. Vickers, J. James, S. Voets, N. Watkins, K. Matthews, P. M, and James. 2007. Anatomically related grey and white matter abnormalities in adolescent onset schizophrenia. Brain.

[9] Kubicki M. Park, H. Westin, C. F. Nestor, P. G. Mulkern, R. V. Maier, S. E., Niznikiewicz, M., Connor, E. E., Levitt, J. J., Frumin, M., Kikinis, R., Jolesz, F. A., McCarley, R. W., and Shenton, M. E. 2005. DTI and MTR abnormalities in schizophrenia: analysis of white matter integrity. Neuroimage.

[10] Sussmann, J. E. Lymer, G. K. McKirdy, J. Moorhead, T. W. Maniega, S. M. Job, D. Hall, J. Bastin, M. E. Johnstone, E. C. Lawrie, S. M. and McIntosh. 2009. White matter abnormalities in bipolar disorder and schizophrenia detected using diffusion tensor magnetic resonance imaging. Bipolar Disord.

[11] Bahn, S. 2002. Gene expression in bipolar disorder and schizophrenia: new approaches to old problems. Bipolar Disord.

[12] Williams, H. J. Norton, N. Dwyer, S. Moskvina, V. Nikolov, I. Carroll, L. Georgieva, L. Williams, N. M. Morris, D. W. Quinn, E. M. Giegling, I. Ikeda, M. Wood, J. Lencz, T. Hultman, C. Lichtenstein, P. Thiselton, D. Maher, B. S. Malhotra, A. K. Riley, B. Kendler, K. S. Gill, M. Sullivan, P. Sklar, P. Purcell, S. Nimgaonkar, V. L. Kirov, G. Holmans, P. Corvin, A. Rujescu, D. Craddock, N. Owen, M. J. and O'Donovan, M. C. 2010. Fine mapping of ZNF804A and genome wide significant evidence for its involvement in schizophrenia and bipolar disorder. Mol. Psychiatry.

[13] Cummings JL, Miller BL. 1987. Visual hallucinations: clinical occurrence and use in differential diagnosis. West J Med.

[14] Bracha HS, Wolkowitz OM, Lohr JB, Karson CN, Bigelow LB. 1989. High prevalence of visual hallucinations in research subjects with chronic schizophrenia. Am J Psychiatry.
[15] A Amad, A Cachia, P Gorwood, D Pins, C Delmaire, B Rolland, M Mondino, P Thomas and R Jardri. 2013. The multimodal connectivity of the hippocampal complex in auditory and visual hallucinations. Molecular Psychiatry.

[16] Calhoun VD, Adali T, Giuliani NR, Pekar JJ, Kiehl KA, et al. 2006. Method for multimodal analysis of independent source differences in schizophrenia: combining gray matter structural and auditory oddball functional data. Hum Brain Mapp.

[17] Specht, K. Zahn, R. Willmes, K. Krause, BJ. Herzog, H. et al. 2008. Tracing the recovery of aphasia with a joint ICA of functional and structural data. 14th Annual Meeting of the Organization of Human Brain Mapping.

[18] Choi, K. Yang, Z. Hu, X. Mayberg, H. 2008. A Combined Functional-Structural Connectivity Analysis of Major Depression Using Joint Independent Components Analysis. Psychiatric MRI/MRS.

[19] João Jorge, Wietske van der Zwaag, Patrícia Figueiredo. 2013. EEG-fMRI integration for the study of human brain function. NeuroImage.

[20] Calhoun, VD. Adali, T. Liu.2006. A feature-based approach to combine functional MRI, structural MRI and EEG brain imaging data. New York City, USA

[21] Eichele T, Calhoun VD, Debener S. 2009. Mining EEGfMRI using independent component analysis. Int $\mathrm{J}$ Psychophysiol.

[22] Calhoun V, Wu L, Kiehl K, Eichele T, Pearlson G. 2011. Aberrant Processing of Deviant Stimuli in Schizophrenia Revealed by Fusion of FMRI and EEG Data. Acta Neuropsychiatr.

[23] Franco AR, Ling J, Caprihan A, Calhoun VD, Jung RE, et al. 2008. Multimodal and Multi-tissue Measures of Connectivity Revealed by Joint Independent Component Analysis. IEEE J Sel Top Signal Process.

[24] Teipel SJ, Bokde AL, Meindl T, Amaro E Jr, Soldner J, et al. 2010. White matter microstructure underlying default mode network connectivity in the human brain. Neuroimage.

[25] Xu L, Pearlson G, Calhoun VD. 2009. Joint source based morphometry identifies linked gray and white matter group differences. Neuroimage

[26] Calhoun VD, Adali T, Giuliani NR, Pekar JJ, Kiehl KA, et al. 2006. Method for multimodal analysis ofindependent source differences in schizophrenia: combining gray matter structural and auditory oddball functional data. Hum Brain Map.

[27] The FIT website. [Online]. Available: http://mialab.mrn.org/software/fit/ [last access, date 27 may 2014]

[28] Xu L, Groth KM, Pearlson G, Schretlen DJ, Calhoun VD. 2009. Source-based morphometry: the use of independent component analysis to identify gray matter differences with application to schizophrenia. Hum Brain Mapp

[29] Liu J, Pearlson G, Windemuth A, Ruano G, PerroneBizzozero NI, et al. 2009. Combining fMRI and SNP data to investigate connections between brain function and genetics using parallel ICA. Hum Brain Mapp. 
[30] Liu, J. Calhoun, VD. 2007. Parallel independent component analysis for multimodal analysis: application to fMRI and EEG data. Arlington, USA.

[31] Wu L, Eichele T, Calhoun VD. 2010. Reactivity of hemodynamic responses and functional connectivity to different states of alpha synchrony: a concurrent EEGfMRI study. Neuroimage.

[32] Liu, J. Pearlson, G. Windemuth, A. Ruano, G. PerroneBizzozero, et al. 2009. Combining fMRI and SNP data to investigate connections between brain function and genetics using parallel ICA. Hum Brain Mapp.

[33] Meda SA, Jagannathan K, Gelernter J, Calhoun VD, Liu J, et al. 2010. A pilot multivariate parallel ICA study to investigate differential linkage between neural networks and genetic profiles in schizophrenia. Neuroimage.

[34] Jamadar S, Powers NR, Meda SA, Gelernter J, Gruen JR, et al. 2010. Genetic influences of cortical gray matter in language-related regions in healthy controls and schizophrenia. Schizophr Res.

[35] Jagannathan K, Calhoun VD, Gelernter J, Stevens MC, Liu J, et al. 2010. Genetic associations of brain structural networks in schizophrenia: a preliminary study. Biol Psychiatry.

[36] J. Liu, O. Demirci, and V. D. Calhoun. 2008. A Parallel Independent Component Analysis Approach to Investigate Genomic Influence on Brain Function. IEEE Signal Proc. Letters. 